\title{
Interpolating Orientation Fields: An Axiomatic Approach
}

\author{
Anatole Chessel ${ }^{1}$, Frederic $\mathrm{Cao}^{2}$, and Ronan Fablet ${ }^{1}$ \\ 1 IFREMER/LASAA \\ BP 70, Technopole Brest-Iroise \\ 28280, Plouzane, France \\ \{achessel,rfablet\}@ifremer.fr \\ 2 IRISA/VISTA \\ Campus de Beaulieu \\ 35041, Rennes, France \\ fcao@irisa.fr
}

\begin{abstract}
We develop an axiomatic approach of vector field interpolation, which is useful as a feature extraction preprocessing step. Two operators will be singled out: the curvature operator, appearing in the total variation minimisation for image restoration and inpainting/disocclusion, and the Absolutely Minimizing Lipschitz Extension (AMLE), already known as a robust and coherent scalar image interpolation technique if we relax slightly the axioms. Numerical results, using a multiresolution scheme, show that they produce fields in accordance with the human perception of edges.
\end{abstract}

\section{Introduction}

Given a set of edgels (i.e. a set of points with an assigned direction), what are the most invariant and stable ways to reconstruct an orientation field in the whole plan? Because orientation live on the unit circle, an everywhere smooth interpolation is not always possible, due to global topological arguments. However, if we now use local arguments, a analysis similar to $[1,2]$ is possible and leads also to similar necessary conditions, showing that only very few differential operators have good properties. Since the functions that will be considered in this paper are vector valued or have values in the unit circle, only little is known about existence, uniqueness or classification of the singularities of the solutions to equations we single out. This contribution is an insight of what could be those results and their interest from a low-level vision point of view.

Detecting what we intuitively call "edges" is a first step towards low level feature extraction and integration and has been the focus of a lot of work since the beginning of computer vision. But as noted by psycho-visual experiment and models [3], that concept has appeared to be more difficult to define than simply "contrasted image part". Psychovision experiments by the Gestaltists [3,4] has given us an acute and unified framework to analyse those effects, and many grouping laws are often involved in the recognition of what we call an edge. The 
so-called subjective contour effect in particular let us see edges which are strictly speaking not even actually present. It uses amodal completion (reconstruction of occluded edges due to the 2D projection of a 3D world) and modal completion (leading to illusory contour, where the object and the background have the same color). In both cases it rely on a curve interpolation process of unknown data according to the input.

The main origin for those subjective contours is the good continuation principle, which states that if two edgels (edge elements, i.e. a collection of points together with the orientation of the curve which should pass through it) are not too far apart and have compatible directions, we tend to see the curve to which they are both tangent as an edge. Many studies have aimed at computationally implementing this phenomenon. To this end, it is generally assumed that a filter has given us an image of edgels from which we want to extract the curves. Two classical approaches are the curve detector of Parent and Zucker [5], which uses a discrete co-circularity measure to extract potentially interesting point, and Sha'ashua and Ullman saliency network [6], where dynamic programming is used to exhaustively search for the "best" curves under curvature minimisation and length maximisation constraints. More recently, interesting approaches are Medioni's tensor voting $[7,8]$, where curves emerge from votes of sparse edgels, and Zweck and al. stochastic completion fields [9], an Euclidean group invariant implementation of the advection-diffusion model of Mumford [10].

Related problems include image inpainting and restoration, and the operators described here are also applied in those cases. In particular, recent developpement extended them to the case of non-scalar image (vector or tensor valued images) [11-13]. The aim however is not exactly the same, as this work does not seek to recover the image itself, but an orientation field that would capture its geometrical features.

The good continuation principle states conditions on tangent vectors, and most of the approaches mentioned earlier rely, explicitly or not, on vector or orientation fields. The present work aims at finding out the most invariant interpolation methods based on partial differential equations (PDE). Experiments will be shown using artificial and natural images.

Section 2 states some generalities about interpolating angle, and in particular that singularities are often unavoidable. Section 3 is devoted to the actual axiomatic approach. The last two sections present in more details the two singled out operators, along with experiments. All the proofs are omitted and are given in [14].

\section{Interpolating Angles}

Let $\Omega \subset \mathbb{R}^{2}$ and $\partial \Omega$ its boundary. Let $S^{1}$ be the unit circle of $\mathbb{R}^{2}$. We consider the extension problem: knowing $I=\left(I_{1}, I_{2}\right): \partial \Omega \rightarrow S^{1} \subset \mathbb{R}^{2}$, how to extend $I$ to the whole domain $\Omega$ ? The circle represents angular data modulo $2 \pi$. In addition, we may also consider the directions of unoriented lines (i.e. angles modulo $\pi$ ). All the argumentation below will also apply to this case. 


\subsection{Topological Restriction}

The first problem we encounter when extending vectors as opposed to scalars, is that singularities in the field may be unavoidable. Given a data to be interpolated when can we hope for a singularity free extension? A necessary and sufficient condition is the following.

Proposition 1. Let $f$ be a continuous vector field over $\partial \Omega$. There exists a continuous extension of $f$ to $\Omega$ if and only if $f$ satisfies condition $\mathrm{C}$.

$$
\exists \alpha \in S^{1}, \alpha \notin f(\partial \Omega),
$$

that is to say only if $f$ is not surjective.

These topological results mean that a singularity free extension is impossible for orientation field when the bounding data cover the whole unit circle. This classical result is equivalent to the Brouwer fixed point theorem [15].

\subsection{A Fundamental Ambiguity}

Another problem arising in orientation interpolation (compared to scalar interpolation) is that, due to the periodicity of the data (hence the absence of a total ordering) there are always two manners to interpolate between two fixed values. For instance, on Fig. 1, one goes through zero, while the other goes through $\frac{\pi}{2}$.

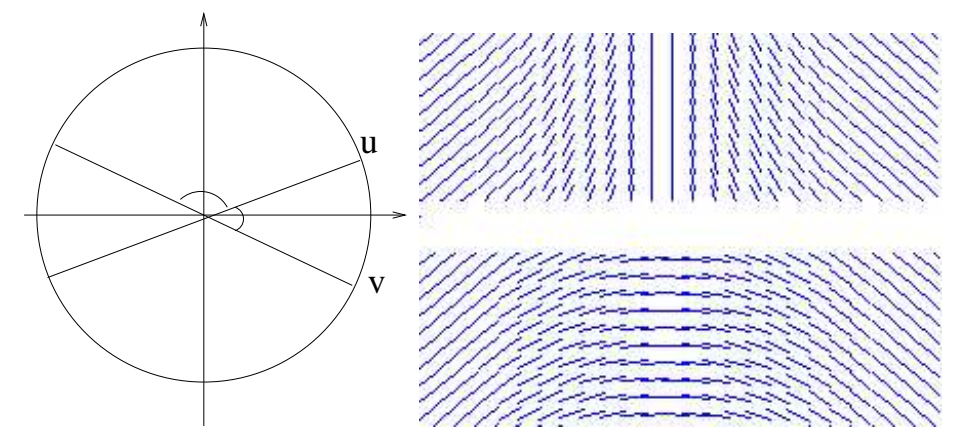

Fig. 1. Ambiguity of interpolation between two directions (modulo $\pi$ in the present figure). Left: there are two ways of going from $u$ to $v$. Right: example when $u-v=\pi / 2$

For a singularity free (non-surjective) field, at least one value is excluded hence only one of the two possibilities is available. The operators we study are numerically solved by iterative methods, hence, in absence of uniqueness result on the solution of the stationary problem, the final result may depend on the initialisation. Moreover, the topological condition (C) might not be fulfilled in practice for the whole domain but only in sub-domains. This could lead to instabilities that do not exist in the scalar case. A possible workaround is a multiresolution scheme, which will be detailed in a forthcoming work [14]. 


\section{Axiomatic Approach}

This section details the axiomatic approach exploited to defined operators for the interpolation of orientation field.

Let $\Gamma$ be a continuous Jordan curve bounding a simply connected domain $\Omega$. We look for an extension operator $E$, which associates with each directional data $\theta_{0}: \Gamma \rightarrow S^{1}$ a unique extension $E\left(\Gamma, \theta_{0}\right)$. Throughout all the discussion to follow, it is assumed that $\theta_{0}$ satisfies the global condition (C). The set of all those functions will be denoted by $\mathcal{F}(\Gamma)$. It is necessary to parameterise $S^{1}$, to be able to deal with numerical functions. A slight difficulty will arise, since it is not possible to describe the whole circle by a unique chart. Let $\phi$ be such a local parameterisation, that is to say, a bijective function $U \rightarrow V$, where $U$ is an open subset strictly included in $S^{1}$ and $V$ an open subset of $\mathbb{R}$. Let us now consider the extension operator $E_{\phi}$ interpolating real valued boundary data $u$, defined by $E_{\phi}(\Gamma, u)=\phi \circ E\left(\Gamma, \phi^{-1} \circ u\right)$. Since $E_{\phi}(\Gamma, u)$ is a numerical function, it is easier to formulate conditions on the operator $E_{\phi}$. However, since the parameterization

$\phi$ is arbitrary, the result should be independent on the parametrisation and condition on $E_{\phi}$ should stand for any $\phi$.

Following [2], we ask that $E_{\phi}$ satisfies the following axioms:

Axiom (A1): Comparison principle Let $\theta_{1}, \theta_{2} \in \mathcal{F}(\Gamma)$ such that they can be described by a common chart $\phi$. Then $\phi\left(\theta_{1}\right) \geq \phi\left(\theta_{2}\right)$ implies

$$
E_{\phi}\left(\Gamma, \phi\left(\theta_{1}\right)\right) \geq E_{\phi}\left(\Gamma, \phi\left(\theta_{2}\right)\right) .
$$

Axiom (A2): Stability principle Let $\Gamma \in \mathcal{C}, \theta_{0} \in \mathcal{F}(\Gamma)$, and $\Gamma^{\prime} \in \mathcal{C}$ such that $D\left(\Gamma^{\prime}\right) \subseteq D(\Gamma)$. Then,

$$
E\left(\Gamma^{\prime},\left.E\left(\Gamma, \theta_{0}\right)\right|_{\Gamma^{\prime}}\right)=\left.E\left(\Gamma, \theta_{0}\right)\right|_{D\left(\Gamma^{\prime}\right)}
$$

Axiom (A3): Regularity principle Let us denote by $D(x, r)$ the disc with center $x$ and radius $r$. Let $Q: \mathbb{R}^{2} \rightarrow S^{1}$ such that there exists a parameterisation $\phi$ such that

$$
\phi(Q)(y)=\frac{1}{2} A_{\phi}(y-x, y-x)+\left(p_{\phi}, y-x\right)+c_{\phi}
$$

where $A_{\phi} \in S M(2)$ the set of two dimensional symmetric matrices, $p_{\phi} \in \mathbb{R}^{2} \backslash\{0\}$, $x \in \mathbb{R}^{2}$ and $c_{\phi} \in \mathbb{R}$. Then there exists a continuous function $F: S M(2) \times$ $\mathbb{R}^{2} \backslash\{0\} \times \mathbb{R} \times \mathbb{R}^{2}$, independent of $\phi$ such that

$$
\lim _{r \rightarrow 0^{+}} \frac{\phi\left(E\left(\partial D(x, r),\left.Q\right|_{\partial D(x, r)}\right)\right)(x)-\phi(Q)(x)}{r^{2} / 2} \rightarrow F\left(A_{\phi}, p_{\phi}, c_{\phi}, x\right) .
$$

Axiom (A4): Translation invariance Let $\tau_{h} \theta_{0}(x)=\theta_{0}(x-h), \theta_{0}: \mathbb{R}^{2} \rightarrow S^{1}$, $h \in \mathbb{R}^{2}$. Then for all $h$,

$$
E\left(\Gamma-h, \tau_{h} \theta_{0}\right)=\tau_{h} E\left(\Gamma, \theta_{0}\right) .
$$

Axiom (A5): Domain rotation invariance For any plane rotation $R$,

$$
E\left(R \Gamma, \theta_{0} \circ R^{-1}\right)=E\left(\Gamma, \theta_{0}\right) \circ R^{-1} .
$$


Axiom (A6): Zoom invariance Let $H_{\lambda} \theta_{0}(x)=\theta_{0}(\lambda x)$, for $\lambda>0$. Then,

$$
E\left(\lambda^{-1} \Gamma, H_{\lambda} \theta_{0}\right)=H_{\lambda} E\left(\Gamma, \theta_{0}\right) .
$$

Once the parametrisation is taken care of, all result obtained in the scalar case are extended to orientation fields. This extension is nearly straightforward, complete proof can be found in [2].

Theorem 1. Assume that the interpolation operator $E$ satisfies (A1)-(A3). Then $F(A, p, x, c)$ does not depend on $c$. Moreover, if $\theta_{0} \in \mathcal{F}(\Gamma)$, then $\phi\left(E\left(\Gamma, \theta_{0}\right)\right)$ is a viscosity solution of

$$
\left\{\begin{array}{l}
F\left(D^{2} u, D u, x\right)=0 \text { in } D(\Gamma) \\
u=\phi\left(\theta_{0}\right) \text { on } \Gamma .
\end{array}\right.
$$

Remark 1. In the scalar case [2], grey scale shift invariance is assumed to prove this result. Of course, it does not make sense since angles do not add. However, since the result must be invariant with respect to the parameterisation, we get an equivalent property for free.

Theorem 2. Assume that $E$ satisfies axioms (1)-(6) and that $F$ is differentiable at 0 . Then, for all parameterisation $\phi, \phi\left(E\left(\Gamma, \theta_{0}\right)\right)$ is solution of

$$
\left\{\begin{array}{l}
D^{2} u\left(D u^{\perp}, D u^{\perp}\right)=0 \text { in } D(\Gamma) . \\
u=\phi\left(\theta_{0}\right) \text { on } \Gamma .
\end{array}\right.
$$

Remark that this operator is the curvature of the level lines of $u$, up to a $|D u|^{3}$ factor. These level lines are independent of the parameterisation, which makes the result possible. Indeed, the independence with respect to parameterisation implies that, for all admissible $\phi$ and $\psi$,

$$
E\left(\Gamma, \theta_{0}\right)=\phi^{-1} \circ E_{\phi}\left(\Gamma, \phi \circ \theta_{0}\right)=\psi^{-1} \circ E_{\psi}\left(\Gamma, \psi \circ \theta_{0}\right) .
$$

By noting $u=\phi \circ \theta_{0}$ and $g=\psi \circ \phi^{-1}$, this equation becomes

$$
g \circ E_{\phi}(\Gamma, u)=E_{\psi}(\Gamma, g \circ u) .
$$

This condition is closely related to invariance with respect to contrast change for scalar data, and the arguments developed in [1] indeed apply.

As noted in disocclusion experiments [16], this operator interpolates the level lines of the data with straight lines. A well known problem is that the solution of this equation may be not unique, and we will see in the next section that if it manages to keep the discontinuities structuring the image, it fails to give a field smooth enough to recover subjective contour. Thus we may drop the full independence over parameterisation and slightly relax Axioms (1) and (3). 
Proposition 2. Assume that Axioms (1) and (3) only holds for parameterisation that are Euclidean, up to a multiplicative factor. Then $\phi\left(E\left(\Gamma, \theta_{0}\right)\right)$ is solution of

$$
a D^{2} u(D u, D u)+b D^{2} u\left(D u, D u^{\perp}\right)+c D^{2} u\left(D u^{\perp}, D u^{\perp}\right)=0,
$$

where $a c-b^{2} \geq 0$.

The condition $a c-b^{2} \geq 0$ ensures that the equation is elliptic, and that the maximum principle can hold. As expected, a solution of (9) is invariant with respect to an affine reparameterisation of the circle, but not to any general parameterisation.

Among all those operators, the case $b=c=0$ is the Absolutely Minimizing Lipschitz Extension (AMLE)

$$
\left\{\begin{array}{l}
D^{2} u(D u, D u)=0 \text { in } D(\Gamma), \\
\left.u\right|_{\Gamma}=\phi\left(\theta_{0}\right) \text { on } \Gamma
\end{array}\right.
$$

for which existence and uniqueness of viscosity solution is known. It gives continuous oscillation free solution. It will be studied in more detail in Sect. 5 .

\section{Angle Interpolation with the Curvature Operator}

As a result of the previous section, the only operator satisfying the given axioms is the curvature operator. It is well known in the computer vision community as a scalar restoration operator via total variation minimisation and has been used for scalar interpolation to solve the disocclusion problem [16-18].

The argumentation above gives the equation which is locally satisfied by the orientation of the vector field. An alternate formulation [19] is to consider the variational problem

$$
\min _{W^{1, p}(\Omega)} \int\|D I\|^{p},
$$

under the constraint $|I|=1$. In this case $I=\left(I_{1}, I_{2}\right)$ and $|I|$ is the Euclidean norm $|I|=\sqrt{I_{1}^{2}+I_{2}^{2}}$ and $\|D I\|=\sqrt{\left|D I_{1}\right|^{2}+\left|D I_{2}\right|^{2}}$. Inspired by the scalar case, we can compute the Euler-Lagrange equations for the energy above by setting $I=\frac{u}{|u|}$ so that the constraint is automatically satisfied. Careful calculations lead to a system of the two coupled PDEs

$$
\operatorname{div}\left(\|D I\|^{p-2} D I_{i}\right)+I_{i}\|D I\|^{p}=0, \quad 1 \leq i \leq 2 .
$$

It is worth noticing that $\|D I\|^{p}$ may be interpreted as the Lagrange multiplier of the constraint $|I|=1$. The case $p=1$, corresponding to the total variation, leads to

$$
\operatorname{div}\left(\frac{D I_{i}}{\|D I\|}\right)+I_{i}\|D I\|=0, \quad 1 \leq i \leq 2 .
$$

As a sanity check, elementary calculations lead to the following result, which holds thanks to the particular choice of norm $\|D I\|$. 
Proposition 3. Let $I=\left(I_{1}, I_{2}\right) \in C^{2}\left(\Omega, \mathbb{R}^{2}\right)$ with $|I|=1$ everywhere. Let $\theta$ such that $I=(\cos (\theta), \sin (\theta))$. Then

$$
\operatorname{div}\left(\frac{D I_{i}}{\|D I\|}\right)+I_{i}\|D I\|=0, \quad 1 \leq i \leq 2 . \Longleftrightarrow \frac{1}{|D \theta|} D^{2} \theta\left(\frac{D \theta^{\perp}}{|D \theta|}, \frac{D \theta^{\perp}}{|D \theta|}\right)=0 .
$$

\subsection{Numerical Resolution}

Experiments (not detailed here) show that the numerical solutions of the two outlined methods are indeed the same. Hence in the following we use the parametrized to $S^{1}$ equation. To compute a numerical solution, we will use the associated evolution problem

$$
\frac{\partial \theta}{\partial t}=D^{2} \theta\left(\frac{D \theta^{\perp}}{|D \theta|}, \frac{D \theta^{\perp}}{|D \theta|}\right)
$$

and let $t \rightarrow \infty$.

To solve this equation, we used a non-linear over relaxation scheme (NLOR) similar to the one found in [2] implemented in Megawave2 [20]. As noted in [21] and [22], we took some special numerical attention to work with angles modulo $2 \pi$ and circumvent the problem caused by the discontinuities at $2 k \pi, k \in \mathbb{N}$. Moreover, as noted above, a multiresolution scheme was used to take care of the initialisation, see [14].

\subsection{Experiments on the curvature operator}

Geometrically, the curvature extension operator tries to extend the level lines of the boundary data by straight lines. Obviously, there are cases for which that approach does not apply [16]. In particular it fails to compute any solution for the simple artificial cases we will see in the next section (see figure 3 ).

However, experiments carried out for larger images with a larger set of boundary points yields interesting results. Figure 2, displays an example with the Lena image. We visualise the field via its field line, using Line Integral Convolution (LIC) [23]. The initial field is given by the orientation of the tangents to the level lines (the orthogonal to the gradient) decimated with a thresholded Canny-Deriche edge filter [24]. As expected, the curvature operator keeps discontinuities, as at the top of Lena's hat. Interestingly enough, it also manages to keep singularities adequately. In particular, T-junctions are preserved, which is particularly relevant in a perceptual point of view. Not only singularities that are present on the boundary data are preserved, but they can also be created in the interpolated area in a suitable way (see for instance at the interface of the cheek and the hair).

To sum up, the curvature operator is (as in the scalar case) able to preserve singularities when necessary. It may be considered as a drawback when the smoothest solution is sought. Moreover, there is no existence and uniqueness result in the general case. 

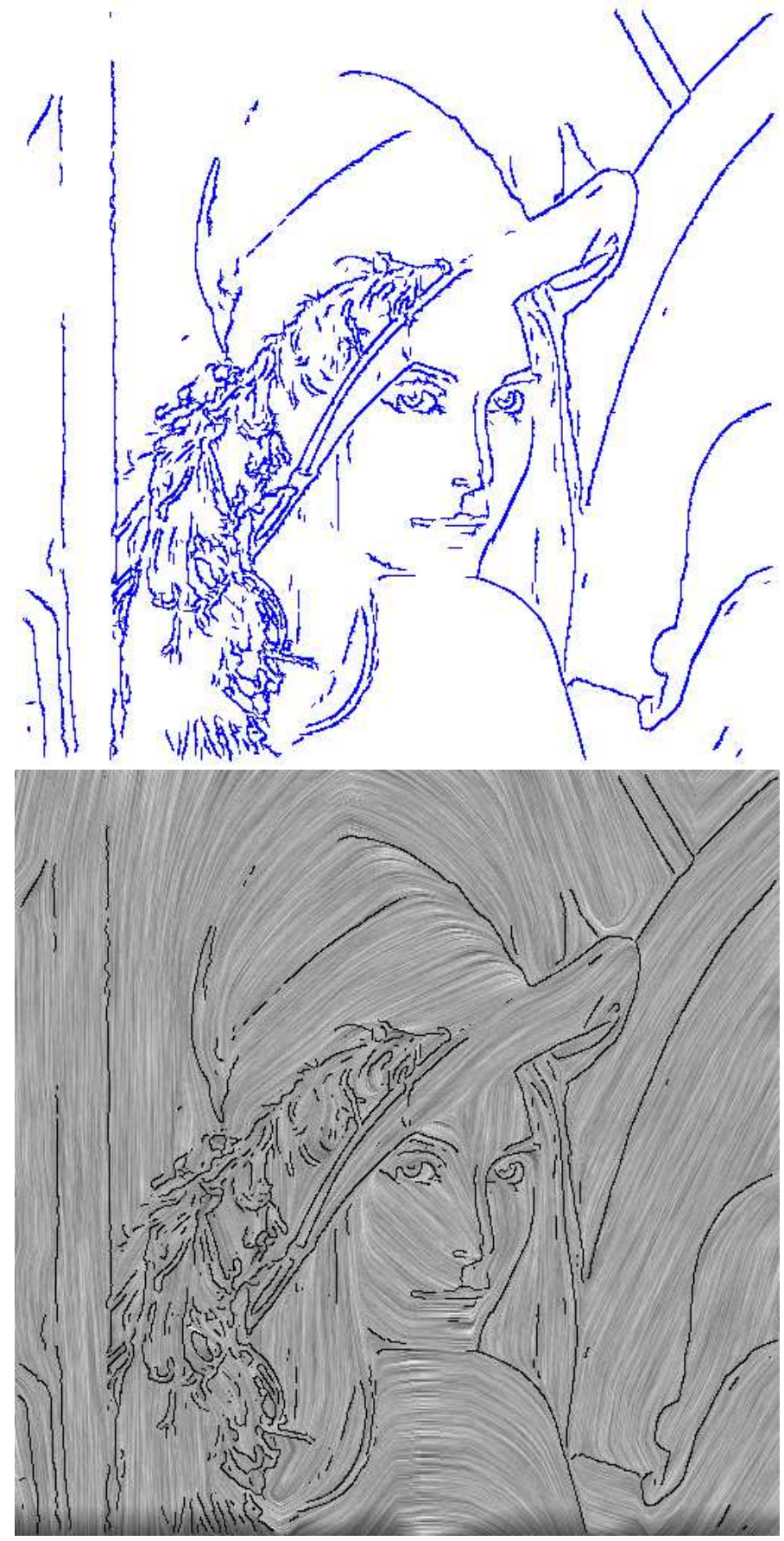

Fig. 2. Interpolation with the curvature operator on Lena. The initialization is orthogonal to the gradient orientation field decimated using a Canny-Deriche filter. A general observation is that T-junctions are preserved. 


\section{AMLE on angle}

In this section, we will provide more insight to the AMLE extension. A more detailed presentation can be found in $[2,25,26]$ We know that a non surjective data can be smoothly interpolated inside a single parameterisation, and that AMLE is independent of affine change of parametrisation.

AMLE was introduced in [27]. It was proved (see [25,26] and references therein) that it can be equivalently defined, in the scalar case, as

- the extension in $\Omega$ of a data defined on $\partial \Omega$ whose Lipschitz constant is minimal in any $\Omega^{\prime} \subset \Omega$.

- the viscosity solution of the PDE $D^{2} u(D u, D u)=0$.

- the limit for $p \rightarrow \infty$ of $p$-harmonic maps, defined as the minimization of the p-harmonic energy

$$
\min _{W^{1, p}(\Omega)} \int|D u|^{p}
$$

Those results heavily rely on a maximum principle (eventually proved by Jensen $[26]$ ), which guarantees in particular that the solution has no oscillation inside the domain. More importantly, it yields the existence and uniqueness of the solution.

\subsection{Equivalence of AMLE on Angle and AMLE on Vector Restricted to $S^{1}$}

Again, we can link the intrinsic formulation on angle used until now and the $\mathbb{R}^{2}$ restricted to $S^{1}$ one. Let us consider (11) again and let $p$ go to $+\infty$. We formally obtain the two coupled equations

$$
\sum_{i=1}^{2} D^{2} I_{i}\left(D I_{i}, D I_{j}\right)=0 \quad j=1,2 .
$$

The definition of a solution of this system is, to the best of our knowledge, an open problem. However, we point out the two following interesting facts.

Proposition 4. Let $I=\left(I_{1}, I_{2}\right) \in C^{2}\left(\Omega, \mathbb{R}^{2}\right)$ with $|I|=1$ everywhere. Let $\theta$ such that $I=(\cos (\theta), \sin (\theta))$. Then

$$
\sum_{i=1}^{2} D^{2} I_{i}\left(D I_{i}, D I_{j}\right)=0 \quad j=1,2 \Longleftrightarrow D^{2} \theta(D \theta, D \theta)=0 .
$$

This means that $I$ is a vector AMLE on the circle if its argument is a scalar AMLE.

The second point is that the term corresponding to the constraint $|I|=1$ has vanished from (11) to (15). Now, a method to solve the stationary problem (15) is to solve the corresponding evolution system

$$
\frac{\partial I_{j}}{\partial t}=\sum_{i=1}^{2} D^{2} I_{i}\left(D I_{i}, D I_{j}\right) \quad j=1,2 .
$$


If $I$ is a continuous solution of (17) such that $|I|=1$ everywhere at time $t=0$, does it remain true for $t>0$ ? At this step, we cannot tell, but we have the following hint.

Lemma 1. Let I be a a $C^{2}$ vector field with $|I|=1$ everywhere. Then the vector with coordinates $\sum_{i=1}^{2} D^{2} I_{i}\left(D I_{i}, D I_{j}\right)$ is everywhere normal to $I$.

\subsection{Numerical Resolution}

Again we can numerically check for the equivalence of the two formulation, and we used the simpler parametrized to $S^{1}$ one. It is proved, in the scalar case, that when $t \rightarrow \infty$ the solution of the evolution problem

$$
\frac{\partial \theta}{\partial t}=D^{2} \theta(D \theta, D \theta)
$$

tends to the solution of the stationary problem, because this solution is unique. In the vector case, we do not have such a result, but we display experiments showing that this is still reasonable. We used a multiresolution NLOR scheme similar to the one used for the curvature operator (see Sect. 4.1).

\subsection{Experiments}

Figure 3 shows numerical results on artificial data. The first one simply consists of two vectors. The interpolated vector field is as expected tangent to the curve with which we would like to connect the two vectors, something close to Euler elastica [10]. The next two figures show the same mechanism with more complex curves: a circle and a tube. The interpolated field is perceptually sound.

As asserted by Prop. 1, we do find singularities in the center of the circle and the extension is there somewhat chaotic, as we are looking for a Lipschitz function where it cannot even be continuous. The situation below the tube (Fig. 3, last experiment) is interesting as it is an example of the ambiguity of Sect. 2.2: a smooth extension do exist, but due to the lack of information the algorithm extended the orientation field the other way round and put a singularity.

We see Fig. 4 an experiment on Lena. The initial field is again the orientation of the tangents to the level lines (the orthogonal to the gradient) decimated with a thresholded Canny-Deriche edge filter [24]. The interpolation field is again tangent to the edges as requested. On the other hand, there is no control on the position of the unavoidable singularities. Moreover, singularities are smoothed out, which can be expected, regarding the properties of the AMLE in the scalar case.

Nonetheless, the AMLE is a good candidate for an interpolation operator as we have a complete theory in the scalar case stating existence and uniqueness of solution. Moreover, it gives smooth solution from which extracting subjective contour as curves is possible. Compared to the curvature however, it tend to lack the ability to keep discontinuities in the fields it produce. 


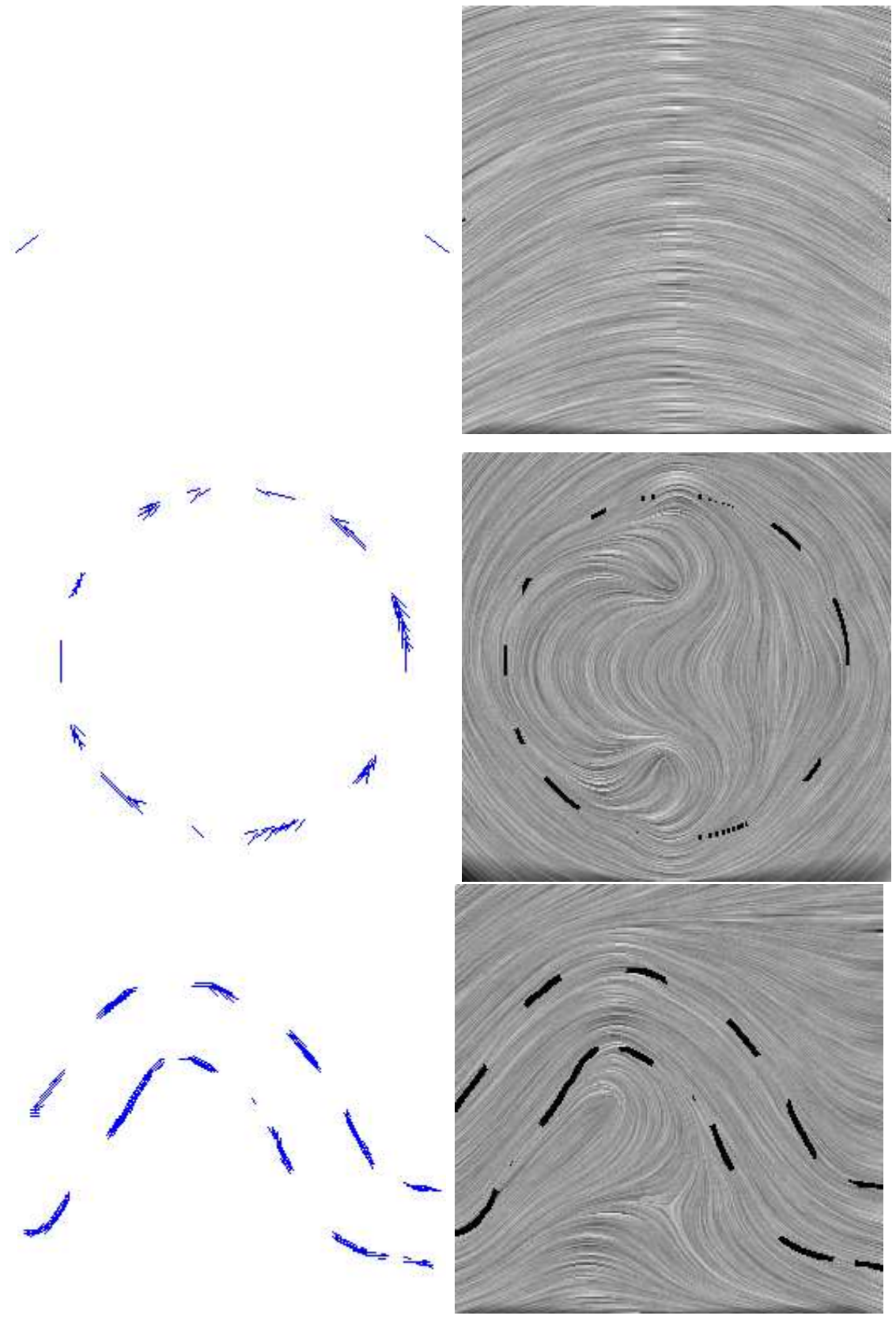

Fig. 3. Three artificial geometric tests, initial orientation field on the left, AMLE extension visualised with LIC on the right. As expected, where we would put a curve it do find the tangent to that curve. It's behaviour is less predictable where there is no information. 

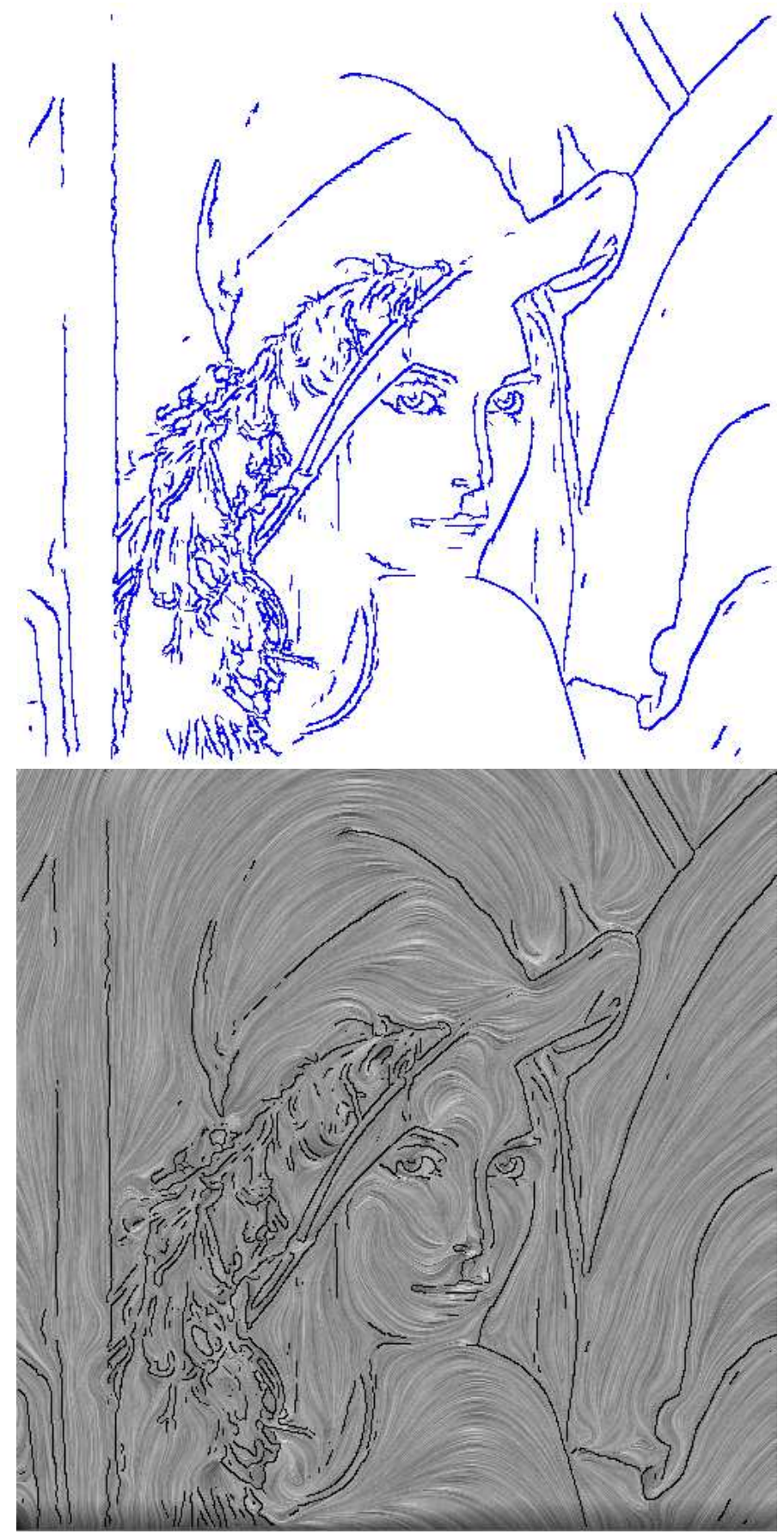

Fig. 4. Test with the Lena image, initialised with the orthogonal to the gradient orientation field decimated using a Canny-Deriche filter. Notice that the recovered field is tangent to the edges, in particular at the top of the hat, on the strands of hair around the face and on Lena's jaw and chin. 


\subsection{Conclusion}

An axiomatic approach of orientation field interpolation has been presented to define extension operators. There is a unique operator satisfying a small set of axioms including geometrical invariance and stability: the curvature operator. This operator is able to preserve singularities. On the other hand, one may require a smoother solution. Moreover, an existence and uniqueness of a solution are not well established. If the independence of the interpolation with respect to reparameterisation of the unit circle is relaxed, another operator becomes interesting: the AMLE. Existence and uniqueness holds in the scalar case. The AMLE is, to some extent, dual to the curvature operator (it minimizes the $L^{\infty}$ norm of the gradient, while the curvature minimizes the norm $L^{1}$ ), and somehow smoothes out the singularities.

The proposed extension operators provides the required basis for the extraction of meaningful curves in images as curves tangent to the orientation field: for instance using Fast Marching approaches [28]. Other obvious applications include LIC-based interpolation or restoration [23, 29]. The operators above are the more natural popping out from the required axioms. However, if some of them are relaxed or more prior knowledge from the image is introduced, some variations of these operators may lead to new types of interpolation model.

\section{References}

1. Alvarez, L., Guichard, F., Lions, P.L., Morel, J.: Axioms and fondamental equations of image processing. Arch. Rational Mechanics and Anal. 16 (1993) 200-257

2. Caselles, V., Morel, J., Sbert, C.: An axiomatic approach to image interpolation. IEEE Trans. Image Processing 7 (1998) 376-386

3. Kaniza, G.: La grammaire du voir. Diderot (1996)

4. Wertheimer, M.: Untersuchungen zur Lehre der Gestalt II. Psychologische Forschung 4 (1923) 301-350

5. Parent, P., Zucker, W.: Trace inference, curvature consistency, and curve detection. IEEE Transactions on Pattern Analysis and Machine Intelligence 11 (1989)

6. Sha'ashua, A., S.Ullman: Structual saliency: The detection of globally salient structures using a locally connected network. In: Second Int. Conf. Comp. Vision, Tarpon Springs, FL. (1988) 321-327

7. Medioni, G., Lee, M.: Grouping .,-,-¿ into regions, curves, and junctions. Computer Vision and Image Understanding 76 (1999) 54-69

8. Medioni, G., Lee, M., Tang, C.: A computational framework for segmentation and grouping. Elsevier Science (2000)

9. Zweck, J., Williams, L.: Euclidian group invariant computation of stochastic completion fields using shiftable-twistable basis function. J. Math. Imaging and Vision 21 (2004) 135-154

10. Mumford, D.: Elastica and computer vision. In Bajaj, C., ed.: Algebraic Geometry and Its Applications. Springer-Verlag (1994)

11. Ballester, C., Caselles, V., J.Verdera: Disocclusion by joint interpolation of vector fields and gray levels. Multiscale Modelling and Simulation 2 (2003) 80-123

12. Kimmel, R., Sochen, N.: Orientation diffusion or how to comb a porcupine. Journal of Visual Communication and Image Representation 13 (2002) 238-248 
13. Osher, S., Sole, A., Vese, L.: Image decomposition and restoration using total variation minimization and the $H^{1}$ norm. Multiscale Modeling and Simulation 1 (2003) 349-370

14. Chessel, A., Cao, F., Fablet, R.: Orientation interpolation : an axiomatic approach. Technical report, IRISA (in preparation)

15. Granas, A., Dugundji, J.: Fixed point theory. Springer-Verlag (2003)

16. Masnou, S., Morel, J.: Level-line based disocclusion. IEEE ICIP (October 1998)

17. Rudin, L.I., Osher, S., Fatemi, E.: Nonlinear total variation bsaed noise removal algorithms. Phisica D 60 (1992) 259-268

18. Chan, T., Shen, J.: Local inpainting model and TV inpainting. SIAM J. Appl. Math. 62 (2001) 1019-1043

19. Tang, B., Sapiro, G., Caselles, V.: Diffusion of general data on non-flat manifolds. Int. J. Computer Vision 36 (2000) 149-161

20. Froment, J.: Megawave2. (http://www.cmla.ens-cachan.fr/Cmla/Megawave/)

21. Perona, P.: Orientation diffusion. IEEE Trans. Image Processing 7 (1998) 457-467

22. Cecil, T., Osher, S., Vese, L.: Numerical methods for minimization problems constrained to $S^{1}$ and $S^{2}$. J. of Computational Physics 198 (2004) 567-579

23. Cabral, B., Leedom, L.: Imaging vector field using line integral convolution. Computer Graphics Proceedings (1993) 263-270

24. Deriche, R.: Using canny's criteria to derive a recursively implemented optimal edge detector. Int. J. Computer Vision 1 (1987) 167-187

25. Aronsson, G., Crandall, M.G., Juutinen, P.: A tour of the theory of absolutely minimizing functions. Bull. Amer. Math. Soc. 41 (2004) 439-505

26. Jensen, R.: Uniqueness of lipschitz extentions: minimizing the sup norm of gradient. Arch. Rational Mechanics and Anal. 123 (1993) 51-74

27. Aronsson, G.: Extention of function satisfying lipschtiz conditions. Ark. Mat. 6 (1997) 551-561

28. Sethian, J.: Level Set Methods and Fast Marching Methods. Cambridge University Press (1999)

29. Tschumperlé, D.: LIC-based regularization of multi-valued images. In: ICIP 05, Genoa, Italy. (2005) 